\title{
XML数据流上的高效聚集算法
}

\author{
王宏志，李建中，骆吉洲
}

(哈尔滨工业大学 计算机科学与技术学院,黑龙江 哈尔滨 150001)

\section{Efficient Aggregation Algorithms on XML Stream}

\author{
WANG Hong-Zhi ${ }^{+}, \quad$ LI Jian-Zhong, LUO Ji-Zhou \\ (School of Computer Science and Technology, Harbin Institute of Technology, Harbin 150001, China) \\ + Corresponding author: E-mail: wangzh@hit.edu.cn
}

Wang HZ, Li JZ, Luo JZ. Efficient aggregation algorithms on XML stream. Journal of Software, 2008,19(8): 2032-2042. http://www.jos.org.cn/1000-9825/19/2032.htm

\begin{abstract}
Each element and value in a XML stream can be accessed only one time. In this paper, efficient algorithms are proposed for processing aggregation on XML streams. These algorithms efficiently support the processing of the aggregation queries with complex structures and the aggregation queries on XML stream with recursion structures. Theoretical analysis and experimental results show that the proposed algorithms are able to process aggregation queries effectively and efficiently on XML stream with high scalability.
\end{abstract}

Key words: XML stream; aggregation; algorithm

摘 要: XML 数据流的特点是所有元素和值仅允许扫描 1 次.针对 XML 数据流上的聚集问题,提出了高效的 XML 数据流聚集算法.这种算法不但能够有效地支持 XML 数据流上具有复杂结构聚集查询的处理,而且能够有效 地支持具有递归结构 XML 数据流上的聚集查询处理.理论分析和实验结果表明,算法能够有效地处理 XML 数据流 上的聚集查询,并且具有很好的可扩展性.

关键词: XML 数据流;聚集;算法

中图法分类号: TP311 文献标识码: A

XML 已经成为因特网上信息表达和交换的重要标准,得到了广泛的应用.XML 数据的查询处理已经引起 了人们的极大关注,取得了很多研究成果.与此同时,人们发现,在很多应用中,如网络信息订阅与发布、电子邮件 监测等, 出现了一类新的数据流, 即 XML 数据流.XML 数据流是一种按照时间顺序实时到达的无限流数据. 由于 XML 数据流仅允许 1 次并按照其数据到达的时间顺序进行存取(不允许多次和随机扫描), XML 数据流的查询 处理成为一个挑战性的研究问题.最近几年, 人们开展了一些相关研究,提出了一些 XML 数据流的查询处理方 法 $^{[1-8]}$. 然而, 目前的研究忽略了一类重要的查询,即 XML 数据流上的聚集查询.下面,我们以电子邮件监测为例 来说明 XML 数据流上的聚集查询的基本思想.

电子邮件可以用 XML 来表示,其简化的模式如图 1 所示,其中 context 是邮件的内容,sender 是发件

* Supported by the National Natural Science Foundation of China under Grant Nos.60773068, 60773063, 60533110 (国家自然科学基 金); the National Basic Research Program of China under Grant No.2006CB303000 (国家重点基础研究计划(973))

Received 2007-09-25; Accepted 2008-04-15 
人,receiver 是收件人.对电子邮件监视系统来说, 电子邮件是源源不断地发出的.因此,电子邮件监视系统面对的 是一个 XML 数据流, 其中每一个邮件是一个 XML 文档片断. 潜在垃圾邮件的判定是电子邮件监视系统的一个 重要功能.一种潜在垃圾邮件的判定方法是:如果系统发现同样内容 的邮件被发送到很多用户,则判定该邮件为潜在垃圾邮件.这种判定 需要对电子邮件流进行如下的聚集查询:“按照所接收的邮件之内容 对邮件接收者分类,计算每个类中接收者的数量.”如果每个类中接收 者数量很大,则表示该类对应的邮件内容被发送到很多用户,相应的 邮件可以被判定为潜在垃圾邮件.上述查询可以表示为电子邮件流 上的聚集查询: //mail/sum(/receiver) with count group by context.在此

$\langle$ !ELEMENT mail (context, sender, receiver*)〉 〈!ELEMENT context \#PCDATA〉 〈!ELEMENT sender \#PCDATA〉 〈!ELEMENT receiver \#PCDATA〉

Fig.1 DTD of E-mail

图 1 电子邮件 DTD 查询中,聚集函数 count 表示首先计算每个 mail 元素中包含 receiver 元素的个数,聚集函数 sum 表示计算每组中 的 mail 元素包含的 message 元素个数的和.group by context 表示 mail 元素需要按照 context 元素进行分组.

XML 数据流上的聚集查询应用十分广泛.下面是另一个 XML 数据流上聚集查询的应用实例. 在基于 XML 的信息定阅 (subscribe)和发布(publish)系统中,XML 数据流是关键,用户常常提出类似于“每天发送的内容与 “database’相关的信息有多少?”、“每个类型的信息发送各有多少?”等查询.这些查询可以表示成聚集查询的 形式.

从上面的讨论不难看出,XML 数据流上的聚集查询是一类应用广泛的重要查询.遗憾的是, 虽然目前已经 提出一些 XML 数据(不是 XML 数据流)上的聚集查询处理算法 ${ }^{[9,10]}$,却一直缺少有关 XML 数据流聚集查询处 理的研究结果. 由于 XML 数据流仅允许 1 次扫描, XML 数据上的聚集查询处理算法无法用来处理 XML 数据流 上的聚集查询,因为这些算法需要多次扫描完整的 XML 数据.

为了有效地处理 XML 数据流上的聚集查询,本文提出了一种高效的 XML 数据流上聚集算法.该算法具有 3 个特点:第一,算法利用栈结构尽早处理聚集的中间结果,减少了中间结果的计算复杂性,提高聚集计算的效率; 第二,针对具有递归结构的 XML 数据流,算法使用栈结构实现了利用后代结点的聚集结果计算祖先结点的聚 集,能够有效地处理具有递归结构的 XML 数据流上的聚集查询; 第三, 算法采用位图、bloom filter、约束矩阵等 技术,解决了 XML 数据流上具有复杂结构聚集查询的有效处理问题. 分析和实验结果表明,本文提出的算法具 有很高的效率和可扩展性, 运行时间是数据量和查询中结点数的线性函数. 本文的主要贡献如下:(1) 本文提出 了 XML 数据流上聚集的问题,并给出了精确定义.(2) 本文提出了高效的 XML 数据流上聚集查询算法,既支持 复杂的 XPath 表达式,又适用于多种类型的 XML 聚集,并且适用于基于 SAX 的 XML 文档上的聚集查询处理.

本文第 1 节给出问题的定义和相关概念. 第 2 节介绍 XML 数据流上的聚集算法及其性能分析. 第 3 节给出 算法的实验结果与分析.第 4 节对本文的内容进行总结.

目前 XML 数据流上查询处理方法主要分为 3 类.第 1 类方法是基于自动机的方法 ${ }^{[1-8]}$.这类方法首先为给 定的 XPath 或 XQuery 查询建立一个自动机, 然后把 XML 数据流作为自动机的输入, 实现查询的处理. 这类方法 没有考虑聚集查询,采用了模式匹配技术实现查询的处理,得到的是未经分组的数据片段,无法处理聚集查询. 第 2 类方法是面向优化的方法 ${ }^{[11,12]}$,重点是通过改写给定的 XQuery 查询,实现查询处理优化. 第 3 类方法 ${ }^{[13-15]}$ 是当查询数量很多时, 对多个查询建立索引, 实现多查询的有效处理. 后两类方法都是对第 1 类方法的扩展, 本质 上仍然基于模式匹配技术,不能处理聚集查询.

关于普通数据流上的聚集查询处理已经有一些技术提出,文献[16]是对这些技术的综述.但是,这些技术不 能用来处理 XML 数据流上的聚集查询. 这是由于 XML 数据流上的聚集操作需要考虑对满足特定路径约束元 素的聚集,传统数据流仅仅考虑数据的聚集而无法对数据的路径约束进行判断.

最近几年, 人们研究了 XML 数据(不是 XML 数据流)上的聚集处理算法 ${ }^{[9,10]}$.文献[9]中提出的聚集算法首先 需要对 XML 文档进行预处理,建立支持聚集的数据结构,然后进行查询处理,要求多次扫描 XML 文档, 与 XML 数据流仅允许 1 次扫描的特点相矛盾, 不能用来有效地处理 XML 数据流上的聚集查询. 文献[10]中提出的方法 用来处理带有约束条件的聚集查询,需要首先在 XML 文档上进行 XPath 查询处理,获得满足约束条件的路径, 
然后在这些路径上进行聚集查询,至少需要扫描 XML 文档两次,因此这种方法不能通过 1 次扫描来处理 XML 数据上的聚集.

\section{1 查询的定义}

XML 文档可以表示为一棵有序、有标签的树,其中,文档中的元素对应树上的结点,文档中元素之间的嵌套 关系对应树上的边. 如果一个元素包含其他元素, 则称这个元素为复合元素. 对于元素 $e$, 从根节点到 $e$ 的路径上 所有元素构成的序列称为 $e$ 的进入路径.XML 数据流可以看作是源源不断到来的 XML 片段,在 XML 数据流上 的处理仅仅允许对每个元素扫描 1 次.

$\mathrm{XPath}^{[17]}$ 表达式用来描述 XML 文档中一类特定的结点,XPath 描述可以分为一系列步骤, 每个步骤均用标 签描述, 步骤间的关系用轴来描述, 在 XPath 标准中定义了 13 种轴, 本文考虑最常见的两种轴:child 和 descendent. 其中,child 表示在 XML 树结构中两个结点之间存在父子关系(简称 PC 关系),用“”来表示; descendent 表示两个结点间存在祖先后代关系(简称 $\mathrm{AD}$ 关系),用“//”来表示.

根据文献[9,10,18]中的定义,XML 上的聚集查询可以表示为如下形式:path ${ }_{a} a / a g g_{-} f_{1} n_{1}\left(\operatorname{path}_{b} b\right)$ with $a g g \_f u n_{2}$ group by path $h_{c_{1}} c_{1}$, path $_{c_{2}} c_{2}, \ldots$, path $_{c_{k}} c_{k}$ using group_fun.其中, $a$ 是聚集操作的对象,称作聚集对象; $b$ 是聚 集对象 $a$ 的一个后代,是聚集操作的具体属性,称作度量属性; $c$ 是用来分组的元素,称为维属性; $p a t h_{a}, p a t h_{b}$ 和 path $_{c_{1}}, \ldots$, path $_{c_{k}}$ 是 XPath 表达式,分别用来修饰元素 $a, b$ 和 $c_{1}, \ldots, c_{k} ; a g g_{-} f u n_{2}$ 是用来对每个聚集对象的度量属性 进行聚集操作的函数,称为内聚集函数; $a g g_{-} f u n_{1}$ 是用来对每个分组中聚集对象的内聚集函数结果进行聚集的 函数,称为外聚集函数;group_fun 是用来定义分组方法的函数,称为分组函数.

XML 文档上的聚集查询与多维数据聚集查询有所不同. 用于分组的元素既可以是简单元素也可以是复合 元素, 而多维数据上聚集查询的分组表示方法不能表达这种复杂的分组,故引入分组函数在分组过程中定义两 个集合或复合元素相等.XML 文档上聚集对象的度量属性可以被多个数据元素匹配,而多维数据聚集查询的度 量属性只能被一条记录匹配 1 次.内聚集函数恰当地表达了这个区别.

聚集查询的结果是一系列元组,每个元组对应一个分组,元组中包含维属性的值和外聚集函数的聚集结果. 一个聚集查询的实例为 $a / b[k] / / c[d[f][g]] / e / \operatorname{sum}(/ / h)$ with count group by $i / j$. 其含义是,对所有满足 XPath 表达式 $a / b[k] / / c[d[f][g]] / e$ 的元素 $e$, 按照 $e$ 后代中满足约束 $i / j$ 的 $j$ 元素进行分组,计算每个分组中 $e$ 的后代中 $h$ 元素的 个数.在这个查询中, $e$ 是聚集对象, $h$ 是度量属性, $j$ 是维属性, count 是内聚集函数, sum 是外聚集函数.

XML 上的聚集查询可以用树结构来表达,查询中的元素表示为树结构中的结点,查询中相邻元素之间的关 系表示为树结构中的边. 在树结构中, path $_{a}$, path $_{b}$, path $_{c_{1}}, \ldots$, path $_{c_{k}}$ 上约束中的结点称为约束结点, path $_{a}$ 中除去约 束结点的其他结点称为主干结点, path $_{b}$, path $_{c_{1}}, \ldots$, path $_{c_{k}}$ 上除去约束结点的其他结点称为中间结点. 对每个结点, 其儿子结点中的约束结点称为该结点的约束结点.查询中的所有结点统称为查询结点.

本文考虑的是 XML 数据流上的聚集查询, 其关键问题是如何在一段时间内对 XML 数据流上多个 XML 片 段中指定的元素有效地进行分组并计算聚集结果.此时,这些 XML 片段可以看作一个具有虚拟根的 XML 文档, 但每个结点只能扫描 1 次.

\section{XML数据流上聚集查询处理算法}

XML 数据流上的聚集查询处理算法包括两部分:一部分是查询对象的获取,得到相应的维属性和度量属 性; 另一部分将获取到的查询对象根据维属性聚集到相应的分组中.这两部分在 XML 数据流处理的过程中交替 进行. 下面,首先给出算法框架, 然后分别给出这两部分的处理方法,最后分析算法的时间复杂性并对算法进行 扩展.

\section{1 算法框架}

为简单起见, 我们仅考虑不包含复合约束条件的查询,带有复杂约束条件聚集查询的处理在第 2.5 节讨论. 聚集查询的处理是两个部分的复合, 一部分是聚集对象的获取, 另一部分是聚集的处理. 前者在数据流中寻 


\section{2 聚集对象获取的优化处理方法}

算法 1 在 XML 数据流中获取查询对象及其对应的维属性和度量属性,它在得到每个结点的全部信息时对 相应的约束进行判断, 这可能会存储过多的中间结点和执行过多的判定. 为加速处理过程和节省内存, 我们给出 聚集对象获取的优化处理方法. 其核心思想是,对每个元素, 尽早地判断该元素满足约束的情况,减少中间结果 数量;对已经满足约束的元素,在处理其后的某些元素时不再对它们是否满足约束进行判定.

由于 XML 数据流只允许 1 次扫描,对于受结构约束的结点, 从当前状态不能判断该元素是否满足所有的查 询条件. 为解决这个问题, 对 v.S 中的每个结点 $v$, 用一个位图 bitmap $v$ 来表示结构约束的满足情况, 其中每个位表 示 $v$ 上的一个特定约束是否被满足. 对不同类型的结点, 其位图有不同结构和约束满足判定方法. 假设元素 $v$ 匹 配查询结点 $q_{v}$, 位图的结构和相应的判定方法如下:

(1) 如果 $q_{v}$ 是约束结点或中间结点, $q_{v}$ 的每个约束对应 bitmap $_{v}$ 的一个位, bitmap $_{v}$ 的长度等于 $q_{v}$ 儿子中约束 结点的个数. 当 $v$ 结点的开始标签到达时, bitmap $v$ 的所有位被初始化为 0 , 这表示 $v$ 上的所有结构约束均未被满 足.在处理过程中, 如果某结构约束被满足, 则 bitmap $_{v}$ 中相应的位将被置 1. 如果 bitmap $v$ 的所有位置为 1, 则表明 $v$ 满足所有的约束条件.

(2) 如果 $q_{v}$ 是主干结点或者聚集对象,任意主干结点的一个结构约束均对应 bitmap $_{v}$ 的一个位, bitmap v $_{v}$ 的长 度等于查询中所有主干结点上结构约束的个数. 当 $v$ 结点的开始标签到达时, bitmap $_{v}$ 中对应主干结点中已被满 足结构约束的位被置为 1 , 其他位被置为 0 . 处理过程中,如果 $v$ 上的某个结构约束被满足, 则 bitmap $v$ 中相应的位 将被置 1; 同时, $v$ 结点所在的路径上的其他主干结点的位图中相应位也将被置为 1. 如果 bitmap $_{v}$ 中对应 $q_{v}$ 上结 构约束的位为 1 , 则表明查询中 $q_{v}$ 结点上的结构约束被满足. 如果 bitmap $_{v}$ 的所有位置为 1 , 则表明 $v$ 结点所在的 路径满足查询中所有的结构约束.

为了加速判定结点 $v$ 上的所有结构约束是否被满足, 我们将判定条件表示成特殊的位图存储在匹配 $v$ 的查 询结点上. 若 $q_{v}$ 是约束结点或者中间结点, 则 $q_{v}$ 上这个特殊位图称为 $q_{v} . L M A P$, 它是一个位数等于 $q_{v}$ 儿子中约束 结点的个数、所有位都是 1 的位图, bitmap $_{v}=q_{v} . L M A P$, 则表明 $v$ 满足 $q_{v}$ 的所有结构约束. 如果 $q_{v}$ 是主干结点或者 聚集对象, $q_{v}$ 上的两个特殊位图分别记作 $q_{v} . L M A P$ 和 $q_{v} . F M A P$, 前者用来判断 $v$ 是否满足 $q_{v}$ 结点上的结构约束, 后者用来判断 $v$ 所在路径是否满足查询中的所有结构约束. 此时, $q_{v} . L M A P$ 是一个位数等于查询中所有主干结点 儿子中约束结点个数的位图, 其中对应 $q_{v}$ 儿子中约束结点的位等于 1 , 其他位为 $0 ; b i t m a p_{v} \& q_{v} . L M A P=q_{v} . L M A P$ 表明 $v$ 满足 $q_{v}$ 结点上的所有结构约束. $q_{v} . F M A P$ 是一个位数与 $q_{v} . L M A P$ 位数相等且所有位都是 1 的位 图, bitmap $_{v}=q_{v} . F M A P$ 表明 $v$ 所在路径满足查询中所有结构约束.

下面讨论位图的更新.在扫描数据流的过程中,如果元素 $e$ 的结束标签到达且 $q_{e}$ 是叶子查询结点,则表明栈 $q_{e}$.parent.S 中与 $q_{e}$.parent 匹配的每个元素 $e^{\prime}$ 对应的结构约束已得到满足,这些元素的位图需要更新.如果 $e^{\prime}$ 的位 图更新后等于 $q_{e^{\prime}} \cdot L M A P$, 表明 $e^{\prime}$ 的所有结构约束均已满足, 则需要进一步更新 $e^{\prime}$ 父亲或祖先的位图, 该过程持续 到遇到根结点或者聚集对象为止. 算法 2 描述了位图更新过程.

算法 2. 路径约束条件的处理.

\section{processBranch(e)}

1 if $q_{e}$ 是叶子 then

2 updateBitmap $\left(q_{e}, e\right)$

updateBitmap (QueryNode $n$, Element $e$ )

1 if $n$ 不是聚集对象或者根结点 then

2 e'=e.parent;

3 bitmap $_{e^{\prime}}[n]=1$
4

5

6

7

8

9

10

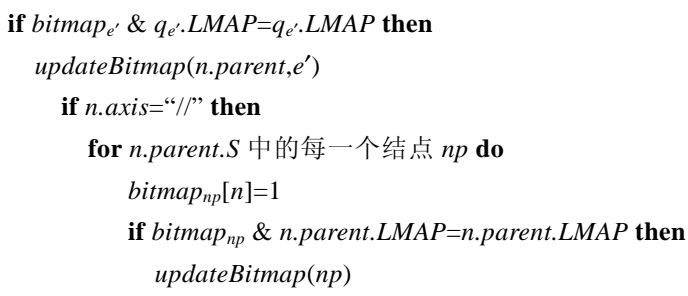

值约束也可以用类似的方法来处理. 若查询结点 $n$ 有值约束, 则在构建查询树时为 $n$ 分配变量 n.value_constraint 以保存这个值约束. 如果 XML 数据流上的元素 $e$ 匹配查询结点 $q_{e}$ 且 $q_{e}$ 有值约束,则当 $e$ 的值 e.value 到达时判断 e.value 是否满足 $q_{e}$.value_constraint. 如果满足, 则置判定变量 e.satisfy 为 true, 否则置为 false. 当元素 $e$ 的结束标签到达时,根据 e.statisfy 的值执行相应的动作. 


\section{3 聚集处理}

本节讨论聚集的处理方法. 聚集过程首先在匹配查询条件的聚集对象上将度量属性按照内聚集函数进行 聚集,再根据维属性对聚集对象进行分组并用外聚集函数聚集. 在该过程中,可以进行如下优化:

(1) 无须等到聚集对象的所有元素到达后才进行聚集.对于聚集对象 $a$,当其度量属性元素 $e$ 的结束标签到 达时,即可将 $e$ 的值用内聚集函数聚集后保存为 $a$ 的临时结果.这样,度量属性的值就无须存储.

(2) 当聚集对象的结束标签到达后, 尽早将其上保存的临时聚集结果用外聚集函数聚集到结果中, 从而节 省该聚集对象上存储这些临时结果的空间.

(3) 当聚集对象存在递归时,后代聚集对象上的聚集结果是其祖先聚集对象上聚集结果的一部分,这部分 聚集结果在后代聚集对象上聚集得到后,无须再在祖先聚集对象上重新计算.这种聚集结果的重用可以提高计 算效率.

为了达到这些优化目标, 中间结点和主干结点需要相应的数据结构.给聚集对象中每个匹配中间结点的元 素 $e$ 赋予变量 $e . v$ 和序列 e.dimension_list,e.v 存储 $e$ 后代中度量属性的临时聚集结果,e.dimension_list 存储 $e$ 的 后代中匹配维属性的元素的值, 称为维序列. 这是为了尽早计算维属性的值和内聚集函数的结果,并丢弃结束标 签已到达的元素. 为每个匹配主干结点的元素 $e$ 赋予序列 e.Agg_list 保存 $e$ 的后代聚集对象. 当 $e$ 的结束标签到 达时,若 $e$ 满足所有约束,则将 e.Agg_list 中的元素合并到结果中; 否则, 将 e.Agg_list 中的元素拷贝到其父亲的 Agg_list 中.此时,释放 $e$ 结点以节省内存.

基于上述优化策略和数据结构,聚集过程在元素 $e$ 的开始标签到达时为 $e$ 分配相应的数据结构.当 $e$ 的结束 标签到达时(此时, $e$ 的所有后代均已处理完毕)根据 $q_{e}$ 的类型和 $e$ 数据结构中的信息执行相应的动作.如果 $q_{e}$ 是维属性, 则将 $e$ 的值加入 e.parent 的维序列. 如果 $q_{e}$ 是度量属性, 则用内聚集函数将 $e$ 的值聚集到 e.parent.v 中.

如果 $q_{e}$ 是中间结点,则需要根据 $q_{e}$ 的轴和位图进行相应的处理. 如果 bitmap $_{e}=q_{e} . L M A P$, 表明 $e$ 及其后代的 结构约束均已被满足,此时, 将 e.v 和 e.dimension_list 合并到 e.parent 的相应数据结构中. 否则, 如果 $q_{e}$ 的轴是“””, 则丢弃 $e$, 因为 $e$ 的所有后代已扫描完毕, $e$ 的条件不可能得到满足;如果 $q_{e}$ 的轴是 “/ ”, 则将 e.v 和 e.dimension_list 拷贝到其祖先上去, 因为尽管 $q_{e}$ 的约束没有被满足, 但 (由于递归) $e$ 的祖先有可能满足条件. 这个过程见算法 3.

如果 $q_{e}$ 是聚集对象, 则根据 $e$ 是否满足结构约束执行相应的动作. 如果 bitmap $_{e}=q_{e}$.FMAP, 则将 e.v, e.dimension_list 和 e.Agg_list 中的元素合并到最终结果去.否则, 如果 $b_{i t m a p} \& q_{e} . L M A P=q_{e} . L M A P$, 则将 $\langle$ e.v, e.dimension_list 拷贝到 e.parent 的 Agg_list 中; 待 e.parent 处理完成后, 根据 e.parent 是否满足结构约束再进行 相应的处理.上述过程正是第 2 个优化策略在聚集对象结点上的具体实现. 在上述过程中, 如果 $q_{e}$ 的轴是 “//”, 则 $e$ 所对应的度量属性和维属性同时也是其祖先聚集对象 $e^{\prime}$ (如果存在, 则在 $q_{e} . S$ 弹出 $e$ 后, $e^{\prime}$ 位于 $q_{e} . S$ 的栈顶)对应 的度量属性和维属性, 因此, 此时还要将e.v 和 e.dimension_list 合并到 $e^{\prime}$ 的数据结构中.这是第 3 个优化策略在聚 集对象结点上的具体实现.聚集对象的处理过程见算法 4.

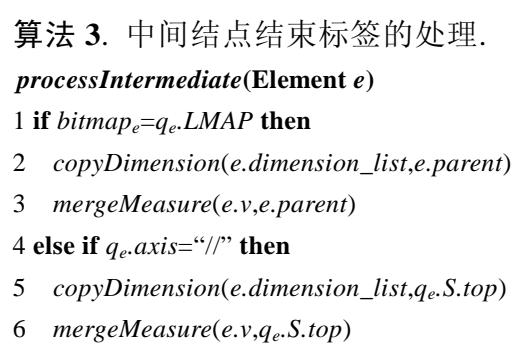

算法 4. 聚集对象结束标签的处理.

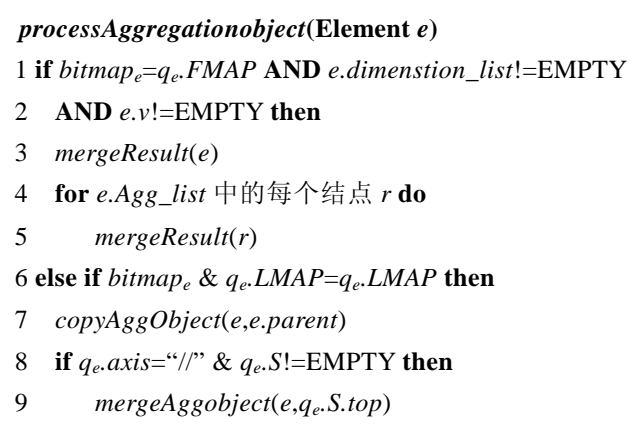

若 $q_{e}$ 是主干结点,则同样根据 $e$ 是否满足结构约束来执行相应的动作.如果 bitmap $_{e}=q_{e}$.FMAP, 则表明 e.Agg_list 中所有聚集对象的路径约束均已被满足, 则将 Agg_list 中的元素用分组函数分组并根据外聚集函数 
束标签到达之前, 如果 $A_{q_{e}}$ 中存在不包含 -1 的列 $a$ 使得 $B_{e} \cdot a=c_{a}$, 则 $e$ 满足 $q_{e}$ 上的复杂约束. 当 $e$ 的结束标签到达 时,如果 $A_{q_{e}}$ 中存在一列 $a$ 使得 $B_{e} \cdot a=c_{a}$, 则 $e$ 满足 $q_{e}$ 上的复杂约束.

由上述命题可知,对于具有复杂约束的查询结点 $q, A_{q}$ 中每列是否出现 -1 和 1 的个数在约束判定过程中起 着重要作用. 为了加速判定过程,这些信息需要抽取出来,存储在 $q$ 对应的数据结构中. 将 $A_{q}$ 每列中由 1 的个数构 成的 $n$ 维整数向量 $C_{q}$ 称为 $q$ 的判定向量. 用 $f_{i}=1(0)$ 表示 $A_{q}$ 中第 $i$ 列是(否)出现 -1 , 向量 $F_{q}=\left\langle f_{1}, . ., f_{n}\right\rangle$ 称为 $q$ 的示性 向量.

当 XML 数据流上元素 $e$ 的开始标签到达时,如果 $e$ 匹配具有复杂约束的查询结点 $q_{e}$, 则根据 $A_{q_{e}}$ 的维数初 始化 $B_{e}$ 为 $m$ 维零向量, 同时初始化 $D_{e}$ 为一个 $n$ 维零向量, $D_{e}$ 的第 $i$ 个分量用来保存当前 $B_{e}$ 与 $A_{q_{e}}$ 的第 $i$ 列的内 积.在处理 $e$ 的某个后代元素时,如果某个原子条件被判断为真,则 $B_{e}$ 中相应的位被置为 1, 同时用 $A_{q_{e}}$ 的相应行 与 $D_{e}$ 相加的结果来更新 $D_{e}$, 然后根据命题结合 $D_{e}$ 、判定向量 $C_{q_{e}}$ 和示性向量 $F_{q_{e}}$ 判断复杂约束是否被满足. 判 定过程见算法 6. 如果此时复杂约束被满足,则在处理 $e$ 的其他后代元素时不再进行判断.如果处理 $e$ 的后代元素 时,复杂约束始终未被满足,则在 $e$ 的结束标签到来时最后进行判断.

算法 6. 根据约束位图判定.

\begin{tabular}{|c|c|c|}
\hline bool changeConstraint(int $k$, Bool is_end) & 4 & $D_{e}[i]=A_{q e}[k][i] * B_{e}[k]+D_{e}[i]$ \\
\hline 1 if $B_{e}[k] !=1$ then & 5 & if $D_{e}[i]=C_{q e}[i]$ AND $\left(f_{i}=0 \|\right.$ is_end $=$ true $)$ then \\
\hline $2 B_{e}[k]=1$ & 6 & return true \\
\hline 3 for $i=1$ to $n$ do & & n false \\
\hline
\end{tabular}

对每个元素 $e$, 向量 $B_{e}$ 的每个分量在整个过程中最多被更新 1 次,只有 $B_{e}$ 的分量被更新时 $D_{e}$ 才会被更新, 每次更新的时间复杂性为 $O(n)$. 所以,处理有复杂约束结点的最坏时间复杂性为 $O(m \times n)$.

\section{3 实验结果}

影响本文提出算法的因素包括 XML 数据流中接收到结点的个数、XML 数据流中 XML 数据片段的最大 高度、查询中约束的个数和维属性的个数. 为了研究本文所提出的算法的性能, 我们从这些方面来对本文提出 的算法进行研究.

\section{1 实验配置}

我们实现了本文提出的 XML 数据流上的聚集算法. 实验的硬件环境是 PC 机,P4 3.0G,1G 内存,150G、7200 转 IDE 硬盘.操作系统是 WindowsXP professional,算法用 Visual C++6.0 实现.

为了有效地测试算法性能,我们采用了 XMark 测试集 ${ }^{[21]}$ 、 DBLP 测 试集 ${ }^{[22]}$, 以及根据图 2 中所示的模式由 XMLGenerator ${ }^{[23]}$ 自动生成的一系列数据 (下文简称为 GD). XMark 数据包含了递归等复杂的结构, 用来测试算法对于多种 聚集查询的效率;DBLP 是真实数据, 用来测试算法在真实数据 上的执行效率;由于在执行带有“//”的聚集查询时,本文所提出的 算法的效率受到 XML 片段高度与递归深度的影响,利用 GD 可 以根据参数控制 XML 片段的高度, 从而测试在不同高度和递归 深度的情况下本文所提出算法的效率. 对于 Xmark, 我们生成了 $10 \mathrm{M} 100 \mathrm{M}$ 的数据; 对于 GD, 我们生成了文档高度为 10 14 的数 据. 我们采用从磁盘上读取 XML 文档、对其进行 1 次扫描的方

$\left\langle\right.$ !ELEMENT $\left.a(b|c| d|e| f|g| h \mid i)^{*}\right\rangle$

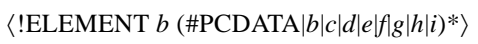
$\left.\langle\text { !ELEMENT c (\#PCDATA }|b| c|d| e|f| g|h| i)^{*}\right\rangle$ $\left.\langle\text { !ELEMENT } d \text { (\#PCDATA }|b| c|d| e|f| g|h| i)^{*}\right\rangle$ $\left.\langle\text { !ELEMENT } e \text { (\#PCDATA }|b| c|d| e|f| g|h| i)^{*}\right\rangle$ $\left.\langle\text { !ELEMENT } f \text { (\#PCDATA }|b| c|d| e|f| g|h| i)^{*}\right\rangle$ $\left.\langle\text { !ELEMENT } g \text { (\#PCDATA }|b| c|d| e|f| g|h| i)^{*}\right\rangle$ $\left.\langle\text { !ELEMENT } h \text { (\#PCDATA }|b| c|d| e|f| g|h| i)^{*}\right\rangle$ 〈!ELEMENT $i$ (\#PCDATA $|b| c|d| e|f| g|h| i) *\rangle$

Fig.2 The DTD of the randomly test data

图 2 随机测试数据的 DTD 式来模拟 XML 数据流. 我们仅仅与用同样方法扫描 1 次 XML 文档但不进行任何操作(在下文中简称 SCAN 操 作)的时间进行比较.

为了有效地测试算法性能, 我们在数据集 XMark 上选取了 8 个查询 $(\mathrm{QX} 1 \sim \mathrm{QX})$, 这组查询覆盖了带有 $\mathrm{PC}$ 和 $\mathrm{AD}$ 关系的查询以及存在各种结构约束的查询;在数据集 DBLP 上选取了 2 个查询 QD1 和 QD2; 在数据集 
GD 上, 选取了 1 个查询 QG. 为了测试查询形态对查询效率的影响, 我们设计了模式相同但参数不同的查询. 具体 的配置将在相应的实验中说明.

\section{2 对比实验}

本小节给出了查询在 XMark100M 数据、DBLP 数据和高度为 15 的 DG 数据上的执行时间和 SCAN 操作 执行时间的比较. 比较结果见表 1. 可以看出, 在 XMark 和 DBLP 数据上执行聚集查询的代价是扫描文档代价的 两倍左右.可见,对于一般的文档和查询, 与扫描相比, 本文所提出的算法具有较高的效率.值得注意的是,算法在 DG 数据集上的执行时间是扫描时间的 4 倍左右, 这是因为其对应的查询树的边全都是 $\mathrm{AD}$ 边且文档存在多层 递归,这些因素均直接影响了算法的效率.

Table 1 Comparison of query execution time and document scan time

表 1 查询执行时间和文档扫描时间的对比

\begin{tabular}{c|ccccccccccc}
\hline Query & QX1 & QX2 & QX3 & QX4 & QX5 & QX6 & QX7 & QX8 & QD1 & QD2 & QG \\
Execution time (ms) & 9656 & 9473 & 10824 & 10246 & 9542 & 9339 & 10829 & 11201 & 28146 & 28226 & 40986 \\
Scan time (ms) & 5683 & 5683 & 5683 & 5683 & 5683 & 5683 & 5683 & 5683 & 13017 & 13017 & 11250 \\
\hline
\end{tabular}

\section{3 可扩展性}

本节给出两组实验, 测试了算法的可扩展性.一组使用 XMark 数据,生成的参数从 0.1 变化到 1 ; 另一组使用 GD, 其生成参数固定结点最大扇出 6,层数从 10 变化到 15.两组实验的结果分别显示在图 3(a)、图 3(b)和图 3(c) 中.可以看出,对 XMark 数据(图 3(a)、图 3(b)), 查询处理时间和文档大小成正比,因此,本文提出的算法具有良好 的扩展性. 对 GD 数据(如图 3(c)所示), 可以看出, 查询处理时间随文档高度的增加呈指数增长, 这是因为文档中 的结点个数随文档高度的增加呈指数增长; 而在相应的查询中, 复杂操作的代价与加法操作类似, 其真实代价和 简单操作相差不多.因此,文档中的结点个数比文档层数对算法效率的影响更大.

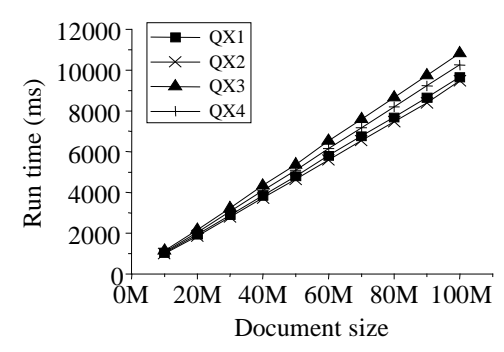

(a) Document size vs. run time (QX1-QX4)

(a) 文档大小 vs. 运行时间(QX1-QX4)

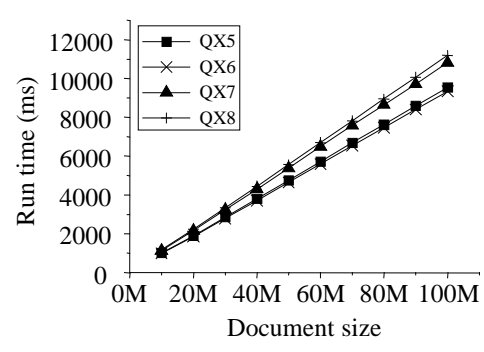

(b) Document size vs. run time (QX5-QX8)

(b) 文档大小 vs. 运行时间(QX5-QX8)

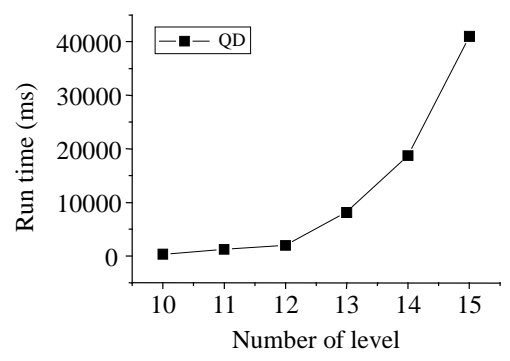

(c) Document height vs. run time (QD)

(c) 文档高度 vs. 运行时间(QD)

Fig.3 Experimental results of scalability

图 3 可扩展性实验结果

\section{4 变化查询参数}

本节通过改变查询中维属性的个数和约束的个数来观察算法运行效率随查询参数变化的趋势. 实验分别 在 XMark100M 和高度为 15 的 GD 数据上运行. 在 XMark 数据集上, 通过改变维属性个数和约束个数分别进行 了两组实验. 在 GD 数据集上进行了一组实验. 算法运行时间随维属性个数变化的结果如图 4(a)所示. 算法运行 时间随约束个数的变化结果如图 4(b)所示. 可以看出, 运行时间随维属性个数和约束个数的增加呈线性增长, 这 符合第 2.4 节中关于算法复杂性的结论, 同时也说明本文提出的算法关于查询结点个数这一参数具有良好的扩 展性.

\section{4 结 论}

本文提出了 XML 数据流上有效的聚集查询处理算法. 本文提出的算法的基本思想是用位图保存数据元素 
满足结构约束的情况,利用栈结构来进行递归情况下“//”轴的处理.本文提出的算法适用于具有复杂结构的聚集 函数和具有递归结构的 XML 数据流. 理论分析和实验结果表明了本文所提出方法的有效性和可扩展性. 除了可 以有效地处理 XML 数据流上的聚集以外,本文提出的算法还适用于在仅允许扫描 XML 文档 1 次的情况下,基 于 SAX 的聚集查询处理.

本文提出的方法是针对单个聚集查询的,在实际应用中,如果可以把多个相关的聚集函数的查询进行合并 处理,则可以提高系统的整体效率.因此,未来的工作包括 XML 数据流上的多聚集查询处理和优化等.

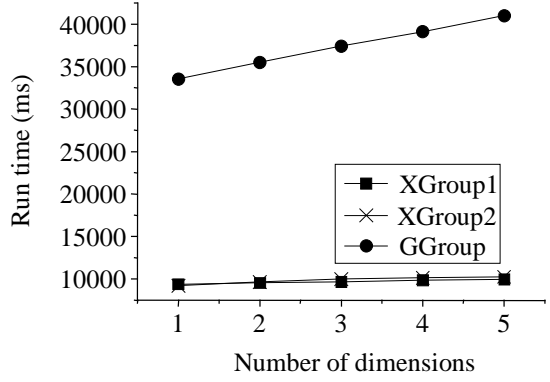

(a) Number of dimensions vs. run time (a) 维属性个数 vs. 运行时间

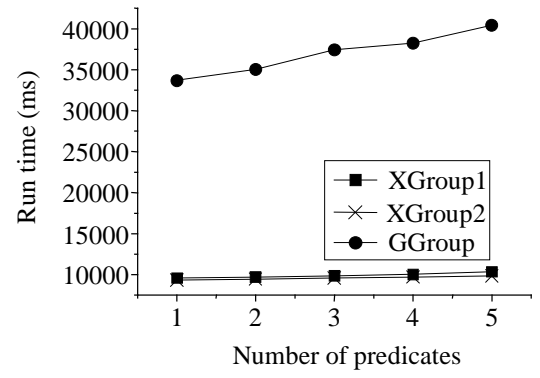

(b) Constraint number vs. run time (b) 约束个数 vs. 运行时间

Fig.4 Experimental result of changing query forms

图 4 改变查询形态的实验结果

\section{References:}

[1] Diao YL, Fischer PM, Franklin MJ, To R. YFilter: Efficient and scalable filtering of XML documents. In: Agrawal R, Dittrich K, Ngu AH, eds. Proc. of the 18th Int'l Conf. on Data Engineering. San Jose: IEEE Computer Society, 2002. 341-344.

[2] Diao YL, Franklin MJ. Query processing for high-volume XML message brokering. In: Freytag JC, Lockemann PC, Abiteboul S, Carey MJ, Selinger PG, Heuer A, eds. Proc. of the 29th Int'l Conf. on Very Large Data Bases. Berlin: Morgan Kaufmann Publishers, 2003. 261-272.

[3] Diao YL, Altinel M, Franklin MJ, Zhang H, Fischer PM. Path sharing and predicate evaluation for high-performance XML filtering. ACM Trans. on Database Systems, 2003,28(4):467-516.

[4] Gupta AK, Suciu D. Stream processing of XPath queries with predicates. In: Halevy AY, Ives ZG, Doan AH, eds. Proc. of the 2003 ACM SIGMOD Int'l Conf. on Management of Data. San Diego: ACM, 2003. 419-430.

[5] Peng F, Chawathe SS. XPath queries on streaming data. In: Nascimento MA, Özsu MT, Kossmann D, Miller RJ, Blakeley JA, Schiefer KB, eds. Proc. of the 30th Int'l Conf. on Very Large Data Bases. Toronto: Morgan Kaufmann Publishers, 2004. $431-442$.

[6] Diao YL, Rizvi S, Franklin MJ. Towards an Internet-scale XML dissemination service. In: Nascimento MA, Özsu MT, Kossmann D, Miller RJ, Blakeley JA, Schiefer KB, eds. Proc. of the 30th Int'l Conf. on Very Large Data Bases. Toronto: Morgan Kaufmann Publishers, 2004. 612-623.

[7] Gao J, Yang DQ, Tang SW, Wang TJ. Tree automata based efficient XPath evaluation over XML data stream. Journal of Software, 2005,16(2):223-232 (in Chinese with English abstract). http://www.jos.org.cn/1000-9825/16/223.htm

[8] Yang WD, Wang QM, Shi BL. Complex twig pattern query Processing over XML streams. Journal of Software, 2007,18(4): 893-904 (in Chinese with English abstract). http://www.jos.org.cn/1000-9825/18/893.htm

[9] Gokhale C, Gupta N, Kumar P, Lakshmanan VS, Ng R, Prakash BA. Complex group-by queries for XML. In: Chirkova R, Dogac A, Ozsu T, Sellis T, eds. Proc. of the 23rd Int'l Conf. on Data Engineering. Istanbul: IEEE Computer Society, 2007. 646-655.

[10] Wang HZ, Li JZ, He YZ, Gao H. Xaggregation: Flexible aggregation of XML data. In: Dong GZ, Tang CJ, Wang W, eds. Proc. of Advances in Web-Age Information Management. Chengdu: Springer-Verlag, 2003. 104-115.

[11] Koch C, Scherzinger S, Schweikardt N, Stegmaier B: Schema-Based scheduling of event Processors and buffer minimization for queries on structured data streams. In: Nascimento MA, Özsu MT, Kossmann D, Miller RJ, Blakeley JA, Schiefer KB, eds. Proc. of the 30th Int'l Conf. on Very Large Data Bases. Toronto: Morgan Kaufmann Publishers, 2004. 228-239. 
[12] Su H, Rundensteiner EA, Mani MM. Semantic query optimization for XQuery over XML streams. In: Böhm K, Jensen CS, Haas LM, Kersten ML, Larson P, Ooi BC, eds. Proc. of the 31st Int'l Conf. on Very Large Data Bases. Trondheim: ACM, 2005. 277-288.

[13] Kwon J, Rao P, Moon B, Lee S. FiST: Scalable XML document filtering by sequencing twig patterns. In: Böhm K, Jensen CS, Haas LM, Kersten ML, Larson P, Ooi BC, eds. Proc. of the 31st Int'l Conf. on Very Large Data Bases. Trondheim: ACM, 2005. 217-228.

[14] Gong XQ, Yan Y, Qian WN, Zhou AY: Bloom filter-based XML packets filtering for millions of path queries. In: Toyama M, Sasaki S, eds. Proc. of the 21st Int'l Conf. on Data Engineering. Tokyo: IEEE Computer Society, 2005. 890-901.

[15] Hou S, Jacobsen HA. Predicate-Based filtering of XPath expressions. In: Liu L, Reuter A, Whang KY, Zhang JJ, eds. Proc. of the 22nd Int'l Conf. on Data Engineering. Atlanta: IEEE Computer Society, 2006. 53-64.

[16] Jin CQ, Qian WN, Zhou AY. Analysis and management of streaming data: A survey. Journal of Software, 2004,15(8):1172-1181 (in Chinese with English abstract). http://www.jos.org.cn/1000-9825/15/1172.htm

[17] Clark J. XML Path language (XPath). W3C, 1999. http://www.w3.org/TR/XPath

[18] Beyer SK, Chamberlin DD, Colby LS, Özcan F, Pirahesh H, Xu Y. Extending xquery for analytics. In: Özcan v, ed. Proc. of the ACM SIGMOD Int'l Conf. on Management of Data. Baltimore: ACM, 2005. 503-514.

[19] Burton H. Bloom. Space/Time trade-offs in hash coding with allowable errors. Communications of the ACM, 1970,13(7):422-426.

[20] Li JZ, Wang S. Principles of Database Systems. 2nd ed., Beijing: Publishing House of Electronics Industry, 2004. 365-366 (in Chinese).

[21] Schmidt A, Waas F, Kersten ML, Carey JM, Manolescu I, Busse R. XMark: A benchmark for XML data management. In: Bressan S, Chaudhri AB, Lee ML, Yu JX, Lacroix Z, eds. Proc. of the 28th Int'l Conf. on Very Large Data Bases. Hong Kong: Morgan Kaufmann Publishers, 2002. 974-985.

[22] Ley M. DBLP DTD. 2001. http://www.acm.org/sigmod/dblp/db/about/dblp.dtd

[23] Diaz AL, Lovell D. XML Generator. 1999. http://www.alphaworks.ibm.com/tech/xmlgenerator

\section{附中文参考文献:}

[7] 高军,杨冬青,唐世渭,王腾蛟. 基于树自动机的 XPath 在 XML 数据流上的高效执行.软件学报,2005,16(2):223-232. http://www.jos.org.cn/1000-9825/16/223.htm

[8] 杨卫东,王清明,施伯乐.针对 XML 流数据的复杂 Twig Pattern 查询处理.软件学报,2007,18(4):893-904. http://www.jos.org.cn/ 1000-9825/18/893.htm

[16] 金澈清,钱卫宁,周傲英.流数据分析与管理综述.软件学报,2004,15(8):1172-1181. http://www.jos.org.cn/1000-9825/15/1172.htm

[20] 李建中,王珊.数据库系统原理.第 2 版,北京:电子工业出版社,2004.365-366.

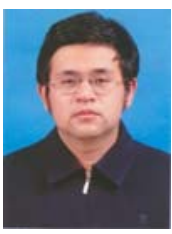

王宏志 $(1978-)$, 男,黑龙江哈尔滨人,博 士,讲师,CCF 学生会员,主要研究领域为 XML 数据管理,信息集成.

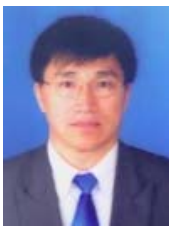

李建中 $(1950-)$, 男, 博士, 教授, 博士生导 师,CCF 高级会员, 主要研究领域为数据库 系统实现技术, 数据仓库, 半结构化数据, 传感器网络, 压缩数据库技术, Web 数据集 成,数据挖掘,计算生物学.

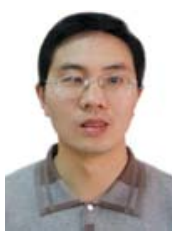

骆吉洲(1975-), 男, 博士, 讲师, 主要研究 领域为压缩数据库. 